$\mathrm{E}$

EVALUAR
2017, Vol. 17, No 2.

ISSN 1667-4545

Recuperado de https://revistas.unc.edu.ar/index.php/revaluar

Laboratorio de Evaluación Psicológica y Educativa

Facultad de Psicología - Universidad Nacional de Córdoba

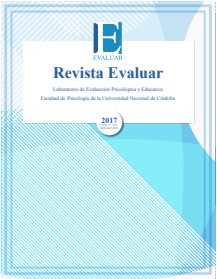

\title{
Propiedades Psicométricas del Inventario de Crecimiento Postraumático en Población Ecuatoriana
}

\author{
Patricio R. Arias * ${ }^{1}$, Felipe E. García ${ }^{2}$, Isabel Patricia Valdivieso ${ }^{3}$ \\ 1 - Universidad Tecnológica Indoamérica, Ambato, Ecuador. \\ 2 - Facultad de Ciencias Sociales, Universidad Santo Tomás, Chile. \\ 3 - Facultad de Ciencias Humanísticas y Sociales, Universidad Técnica de Manabí, Portoviejo, Ecuador.
}

\author{
Introducción \\ Metodología \\ Resultados \\ Discusión \\ Referencias
}

Recibido: 11/07/2017 Revisado: 01/08/2017 Aceptado: 15/08/2017

\section{Resumen}

Dentro del estudio del comportamiento humano ante la exposición a un evento altamente estresante, la psicología ha dado un salto al estudiar no sólo sus efectos negativos sino también el aprendizaje que la persona puede extraer de dichas vivencias, lo que se denomina crecimiento postraumático. El objetivo de este estudio fue analizar las propiedades psicométricas del Inventario de Crecimiento Postraumático (PTGI) en una muestra de 450 adultos sobrevivientes al terremoto ocurrido en el año 2016 en las costas de Ecuador. El PTGI reveló una alta consistencia interna $(\alpha=.95)$. El análisis factorial confirmatorio mostró un ajuste apropiado para una estructura bifactor de tres factores específicos y un factor general. El PTGI además mostró invarianza factorial al comparar hombres y mujeres. Se concluye que el PTGI es un instrumento confiable y con validez interna para investigar el crecimiento postraumático en población ecuatoriana.

Palabras Clave: psicología positiva, análisis factorial confirmatorio, desastre natural, terremoto

\begin{abstract}
In the study of human behavior concerning the exposition to highly stressful events, psychology has taken a leap forward, studying not only the negative effects of such events, but also the knowledge a person may gain from them, which is known as posttraumatic growth. The aim of this study was to analyze the psychometric properties of the Posttraumatic Growth Inventory (PTGI) in a sample of 450 adult survivors of the earthquake that took place on the Ecuadorian coast in 2016. The PTGI showed high internal consistency $(\alpha=.95)$. The confirmatory factor analysis displayed an appropriate adjustment for a bifactor structure consisting of three specific factors and one general factor. Furthermore, the PTGI showed a factorial invariance when comparing men and women. We could conclude that the PTGI is a reliable and internally valid instrument for the study of posttraumatic growth in Ecuadorian population.
\end{abstract}

Keywords: positive psychology, confirmatory factorial analysis, natural disaster, earthquake 


\section{Introducción}

Un sobreviviente a un evento en el que ha corrido riesgo su vida, la de personas cercanas o que ha presenciado este riesgo en otras personas puede llegar a sufrir síntomas de estrés postraumático (American Psychiatric Association, 2013); sin embargo, muchos de ellos son capaces de percibir cambios positivos luego de estos eventos (Tedeschi \& Calhoun, 1996, 2000), lo que les permite alcanzar un mayor bienestar y ajuste psicológico y controlar a largo plazo la aparición de trastornos mentales (García, Cova-Solar, \& Melipillán, 2013). A este fenómeno se lo ha denominado crecimiento postraumático (CPT). El objetivo principal del presente estudio fue evaluar las propiedades psicométricas de un instrumento que permita medir estos cambios en población ecuatoriana afectada por un desastre natural.

El CPT abarca tres dimensiones conceptuales: a) cambios en la autopercepción, que incluyen sentirse más fuerte, seguro y capaz de afrontar dificultades en el futuro; b) cambios en la relación con otros, que incluyen mayor cercanía con la familia y amistades y mayor apertura para recibir ayuda, y c) cambios en la filosofía de vida, que se define como una mayor valoración de la propia existencia e implica tomar la vida con más calma y disfrutar el momento (Tedeschi \& Calhoun, 2008; Calhoun, Cann, \& Tedeschi, 2010). Estas tres dimensiones teóricas no se han traducido necesariamente en una cantidad equivalente de factores cuando se ha evaluado el instrumento diseñado para medir estos cambios: el Inventario de Crecimiento Postraumático (PTGI, por sus siglas en inglés).

El PTGI es un instrumento que consta de 21 ítems y que mostró en su versión original poseer una alta consistencia interna $(\alpha=.90)$ y estabilidad temporal $(r=.71)$. A través de un análisis factorial exploratorio se determinó que estaba cons- tituido por cinco factores (Tedeschi \& Calhoun, 1996): 1) relación con otros, incluye ítems acerca del fortalecimiento de las relaciones interpersonales, (2) apreciación de la vida, incluye ítems acerca del aumento del valor que la persona da a la vida, (3) fuerza personal, incluye ítems acerca de una mejor percepción de las propias capacidades, habilidades y resistencia personal, (4) cambio en la filosofía de la vida, incluye ítems sobre la reevaluación del sentido y significado de la vida tras la experiencia traumática y (5) nuevas posibilida$d e s$, incluye ítems acerca del aumento en la sensación de que la vida brinda nuevas oportunidades $\mathrm{y}$ prioridades.

El PTGI se ha utilizado para trabajar con poblaciones que han vivido diversos eventos traumáticos tales como desastres naturales (García, Jaramillo, Martínez, Valenzuela, \& Cova-Solar, 2014), violencia (Hooper, Marotta, \& Depuy, 2009), diagnósticos de enfermedades que amenazan la vida (Brunet, McDonough, Hadd, Crocker, \& Sabiston, 2010), guerras (Palmer, Graca, \& Occhietti, 2012) o eventos diversos (García, Páez, Reyes-Reyes, \& Álvarez, 2017). Este instrumento ha llegado a ser el más utilizado para el estudio de los efectos positivos de una vivencia potencialmente traumática (Joseph \& Linley, 2008).

Las adaptaciones realizadas al PTGI no siempre han mostrado la estructura interna de los cinco factores que encontraron Tedeschi y Calhoun (1996). Además, se han encontrado estructuras que varían desde uno a cuatro factores, entre ellas la estructura de tres dimensiones que propone el modelo teórico original, como se puede observar en la Tabla 1.

En el análisis de las propiedades psicométricas del PTGI de Joseph, Linley y Harris (2004) se consideró un modelo de un solo factor por la fuerte relación entre sí que habían mostrado los cinco factores iniciales, sin embargo los autores aceptaron la posibilidad de que el instrumento 
Tabla 1

Estructuras factoriales encontradas en el PTGI.

\begin{tabular}{|c|c|c|c|}
\hline Estructura factorial & Autor & País & Muestra \\
\hline \multirow[t]{2}{*}{ Un factor } & Joseph et al. (2004) & Reino Unido & $\begin{array}{l}176 \text { pacientes que han experimentado } \\
\text { acontecimientos angustiosos de la vida }\end{array}$ \\
\hline & $\begin{array}{l}\text { Costa-Requena } \\
\& \text { Gil-Moncayo } \\
(2007)\end{array}$ & España & 130 pacientes con diagnóstico de cáncer \\
\hline \multirow[t]{2}{*}{ Tres factores correlacionados } & Powell et al. (2003) & $\begin{array}{l}\text { Sarajevo, } \\
\text { Bosnia y } \\
\text { Herzegovina }\end{array}$ & $\begin{array}{l}136 \text { adultos refugiados y desplazados } \\
\text { expuestos a diversos eventos } \\
\text { traumáticos }\end{array}$ \\
\hline & $\begin{array}{l}\text { Anderson \& } \\
\text { Lopez-Baez (2008) }\end{array}$ & EE.UU. & 345 estudiantes universitarios \\
\hline $\begin{array}{l}\text { Modelo jerárquico de tres factores } \\
\text { de primer orden y uno de segundo } \\
\text { orden }\end{array}$ & García et al. (2013) & Chile & 449 sobrevivientes a un desastre natural \\
\hline $\begin{array}{l}\text { Modelo bifactor de cinco factores } \\
\text { específicos y un factor general }\end{array}$ & $\begin{array}{l}\text { Konkolÿ-Thege et } \\
\text { al. (2014). }\end{array}$ & Hungría & $\begin{array}{l}691 \text { personas que experimentaron algún } \\
\text { tipo de trauma o pérdida }\end{array}$ \\
\hline \multirow[t]{2}{*}{$\begin{array}{l}\text { Modelo bifactor de tres factores } \\
\text { específicos y un factor general }\end{array}$} & $\begin{array}{l}\text { Rodríguez-Rey et } \\
\text { al. (2016) }\end{array}$ & España & 143 padres de niños hospitalizados \\
\hline & $\begin{array}{l}\text { Garrido-Hernansaiz } \\
\text { et al (2017) }\end{array}$ & España & 304 adultos seropositivos \\
\hline \multirow{6}{*}{$\begin{array}{l}\text { Modelo de cinco factores } \\
\text { correlacionados }\end{array}$} & Brunet et al. (2010) & Canadá & 470 pacientes con cáncer \\
\hline & $\begin{array}{l}\text { Esparza-Baigorria } \\
\text { et al. (2016) }\end{array}$ & Argentina & 156 pacientes oncológicos \\
\hline & $\begin{array}{l}\text { Hooper et al. } \\
(2009)\end{array}$ & EE.UU. & 143 estudiantes parentalizados \\
\hline & $\begin{array}{l}\text { Palmer et al. } \\
(2012)\end{array}$ & EE.UU & 221 veteranos de guerra \\
\hline & $\begin{array}{l}\text { Garcia da Silva et } \\
\text { al. (2016) }\end{array}$ & Brasil & 300 estudiantes universitarios \\
\hline & Taku et al. (2008) & Varios & $\begin{array}{l}926 \text { personas expuestas a distintos } \\
\text { eventos traumáticos }\end{array}$ \\
\hline
\end{tabular}

incluyera tres factores. De la misma manera, utilizando un análisis exploratorio de componentes principales (ACP) se encontraron tres factores tanto en estudiantes universitarios (Anderson \& López-Báez, 2008) como en sobrevivientes de guerra (Powell, Rosner, Butollo, Tedeschi, \& Calhoun, 2003); estos dos estudios conservaron los factores de cambio en la relación con otros y de filosofía de la vida, pero nuevas posibilidades, apreciación de la vida y fuerza personal se reunieron en un solo factor al que llamaron cambios en la autopercepción. Con el análisis factorial confirmatorio del PTGI traducido al español se obtuvo en Chile esa misma estructura de tres factores de primer orden y un factor de segundo orden (García et al., 2013) en un estudio con per- 
sonas adultas que fueron afectadas por el terremoto del año 2010. A su vez, en Argentina se ha encontrado la estructura original de cinco factores correlacionados (Esparza-Baigorria, Leibovich de Figueroa, \& Martínez-Terrer, 2016) en mujeres adultas afectadas por cáncer de mama. Por otra parte, Rodríguez-Rey, Alonso-Tapia, KassamAdams y Garrido-Hernansaiz (2016) y GarridoHernansaiz, Rodríguez-Rey y Alonso-Tapia (2017) obtuvieron en España, con una versión de 12 y 11 ítems respectivamente, un modelo bifactor con tres factores específicos: cambios en la filosofía de la vida, cambio en la autopercepción y cambios en la relación con otros, y un factor general, utilizando la traducción para población hispana de los EE.UU (Weiss \& Berger, 2006).

En el estudio con diversas poblaciones que utilizó el PTGI ha llamado la atención la diferencia de género, de modo que las mujeres informan mayor CPT que los hombres. Un metaanálisis que incluyó 70 estudios llevó a la conclusión de que en el CPT existen diferencias de género modestas, pero significativas entre hombres y mujeres, siendo las mujeres las que muestran más crecimiento que los hombres (Vishnevsky, Cann, Calhoun, Tedeschi, \& Demakis, 2010). Esta diferencia se ha observado también en población latinoamericana (García, Capponi, et al., 2016; García, Jaramillo, et al., 2014).

En el caso de Ecuador, al ser un país expuesto a constantes riesgos de origen natural, la adaptación del PTGI puede contribuir al estudio de las respuestas psicológicas frente a las amenazas, lo que permitirá estudiar los cambios positivos que los ecuatorianos sobrevivientes al terremoto de abril de 2016 han presentado tras el sismo. De ese modo, el objetivo del presente estudio es evaluar las propiedades psicométricas de una traducción del PTGI hecha para población ecuatoriana, para lo cual se evaluará su estructura factorial y la invarianza factorial entre grupos de hombres y mu- jeres, además de los estadísticos descriptivos y la consistencia interna del instrumento. Hasta hoy, no se han realizado estudios que hayan utilizado esta escala en Ecuador, por lo que este sería el primer estudio que evalúe sus propiedades psicométricas en esta población.

\section{Metodología}

Diseño

El diseño utilizado tiene un carácter no experimental, es de naturaleza descriptiva y transversal y es de índole instrumental.

\section{Participantes}

Participaron 450 individuos, 256 mujeres (56.9\%) y 194 hombres (43.1\%). La edad promedio fue de $26.7(\mathrm{DE}=10.66)$ con un rango de 18 a 65 años. Los participantes son pobladores sobrevivientes al terremoto del 16 de abril del año 2016 en la provincia de Manabí.

\section{Instrumentos}

Se utilizó el Inventario de Crecimiento Postraumático (PTGI; Tedeschi \& Calhoun, 1996), el cual consta de 21 ítems que se responden en una escala Likert desde 0 (Ningún cambio) hasta 5 (Un cambio enorme). Se realizó una traducción y traducción inversa para obtener una versión adecuada para la población ecuatoriana (ver Anexo). Las propiedades psicométricas del instrumento en el presente estudio se detallan en la sección de Resultados. Además se incluyó un cuestionario socio-demográfico breve en el que se registró el sexo, la edad y el nivel de estudio de los participantes. 


\section{Procedimiento}

En primer lugar se realizó una nueva traducción del instrumento distinta a las ya disponibles con la finalidad de evitar modismos lingüísticos de otros países, considerando que la conformación multiétnica y multicultural ecuatoriana podría implicar construcciones lingüísticas diferentes. Para ello, un experto psicólogo que maneja los dos idiomas realizó una traducción directa de la escala original del inglés al español, luego otro psicólogo experto realizó una traducción inversa para comparar las coincidencias lingüísticas. El resultado final se sometió a juicio experto con un investigador que ha estudiado el CPT en otra población latinoamericana y se aplicó en una prueba piloto con 70 estudiantes universitarios que fueron afectados por el terremoto, quienes verificaron la comprensión de las instrucciones y de los ítems. Un análisis de consistencia interna arrojó buenos resultados ( $\alpha=.95$ para la escala total).

Para el cálculo del tamaño muestral adecuado para la población objetivo se siguió el criterio recomendado por Martínez-Arias (1995), quien sugiere un mínimo de 300 participantes o entre 5 y 10 por ítem. Siguiendo este último criterio, y dado que el PTGI consta de 21 ítems, la muestra óptima debía incluir un mínimo de 210 participantes. Finalmente se encuestó a 450 personas, lo que permitió que los análisis presentados tuvieran suficiente potencia.

El estudio se realizó en la población de la provincia de Manabí sobreviviente al terremoto del 16 de abril de 2016, para lo cual se solicitó la colaboración de docentes de una universidad de la zona, a quienes se capacitó para la aplicación del instrumento. Ellos a su vez solicitaron a sus estudiantes que lo aplicaran a personas de su contexto social cercano.

Antes de responder al instrumento, se pidió a los participantes firmar un formulario de con- sentimiento informado en el que se aseguraba el anonimato, la confidencialidad de los datos, la voluntariedad para responder y el resguardo de los datos por parte de los investigadores.

\section{Análisis de datos}

Se realizó un análisis factorial confirmatorio (AFC) poniendo a prueba cinco modelos: el primero de ellos fue el modelo de un factor (Joseph et al., 2004); el segundo fue el modelo de tres factores jerárquico (García et al., 2013) que, debido a que se encontró en población latinoamericana también afectada por un terremoto, constituyó el modelo principal evaluado en este estudio; el tercero fue el modelo bifactor de tres factores específicos y un factor general, encontrado en recientes estudios con población española (GarridoHernansaiz et al., 2017; Rodríguez-Rey et al., 2016); el cuarto fue el modelo de cinco factores jerárquico, equivalente al obtenido en Argentina (Esparza-Baigorria et al., 2016) y finalmente se evaluó un modelo bifactor de cinco factores específicos y un factor general, encontrado en una muestra húngara (Konkolÿ-Thege, Kovács, \& Balog, 2014).

Para los análisis se utilizó el método de estimación de máxima verosimilitud, previa evaluación de la normalidad multivariante a través del coeficiente de Mardia (1970, 1974); existe normalidad cuando los valores se ubican dentro del rango \pm 5 (Bentler, 2005) pero se considera adecuado utilizar el método de estimación de máxima verosimilitud cuando los valores no exceden el rango \pm 70 (Rodríguez-Ayán \& Ruiz-Díaz, 2008). La calidad del ajuste de los modelos analizados se evaluó en base a los siguientes estadísticos y criterios de buen ajuste (Byrne, 2006; Kline, 2005): a) $\chi^{2}$ : un valor no significativo se considera un ajuste adecuado; b) $\chi^{2} / g l$ : un valor menor a 3 indi- 
Tabla 2

Índices de ajuste de los modelos.

\begin{tabular}{lccccccc}
\hline \multicolumn{1}{c}{ Modelo } & \multicolumn{1}{c}{$\chi^{2}(\boldsymbol{g l})$} & $\chi^{2}(\boldsymbol{g l})$ & CFI & TLI & AIC & SRMR & $\begin{array}{c}\text { RMSEA } \\
\mathbf{( 9 0 \% ~ I C )}\end{array}$ \\
\hline 1: Un factor & $805.02^{* * *}(189)$ & 4.26 & .90 & .89 & 931.02 & .04 & $.09(.08 / .09)$ \\
2: Tres factores-jerárquico & $579.17^{* * *}(186)$ & 3.11 & .93 & .94 & 711.17 & .04 & $.07(.06 / .08)$ \\
3. Tres factores-bifactor & $365.83^{* * *}(165)$ & 2.22 & .97 & .96 & 497.83 & .03 & $.05(.05 / .06)$ \\
4: Cinco factores-jerárquico & $594.07^{* * *}(184)$ & 3.18 & .93 & .94 & 730.07 & .04 & $.07(.06 / .08)$ \\
5. Cinco factores-bifactor & $351.58^{* * *}(158)$ & 2.23 & .97 & .96 & 497.58 & .03 & $.05(.05 / .06)$ \\
\hline
\end{tabular}

Nota. ${ }^{* * *} p<.001$

ca buen ajuste y aceptable cuando es menor de 5;

c) CFI y TLI: un valor .95 o superior es indicador de un ajuste adecuado, un valor sobre .90 indica un ajuste aceptable; d) AIC: es un índice comparativo, por lo que el modelo que presente el valor más bajo es el que mejor se ajusta a los datos; e) SRMR: un valor inferior a .08 es un ajuste aceptable; f) RMSEA e intervalo de confianza del 90\%: un valor inferior a .08 es un ajuste aceptable, el intervalo de confianza debe tener menos que un .10 en su valor superior.

Se realizó una comparación de grupos a través de $t$ de Student para ver si existían diferencias por sexo. Luego se estudió la invarianza factorial entre los participantes hombres y mujeres a través de un análisis multi-muestra. Para ello, se realizó la evaluación secuencial de la invarianza configural, métrica, fuerte y estricta (Elosúa, 2005). La invarianza configural implica que los factores están especificados por los mismos ítems en los dos grupos. La invarianza métrica evalúa la igualdad de coeficientes de regresión. La invarianza fuerte evalúa la igualdad en los interceptos. La invarianza estricta evalúa la igualdad en los errores y representa el mayor nivel de acuerdo alcanzable entre estructuras factoriales.

Tradicionalmente, la prueba de invarianza entre niveles se realiza estudiando las diferen- cias en el estadístico $\chi^{2}$ entre un nivel y otro, ya que los modelos se encuentran anidados (Bollen, 1989). Como este estadístico es sensible al tamaño de la muestra, es probable que se rechace la invarianza en muestras grandes, por lo que se consideró principal indicio de invarianza que el CFI no varíe más de .01 con respecto al modelo anterior (Cheung \& Rensvold, 2002). Se espera además un TLI superior a $.90 \mathrm{y}$ un RMSEA inferior a .08 .

Se empleó el estadístico alfa de Cronbach para estimar la consistencia interna del PTGI total y de sus factores, así como la correlación ítem-total corregida. Por último, se realizó un análisis descriptivo del instrumento y sus factores, así como una evaluación de la correlación bivariada entre ellos. Los análisis se llevaron a cabo empleando el programa SPSS v.21 (IBM Corp., 2011) y AMOS SPSS v.20. (Arbuckle, 2011)

\section{Resultados}

Para realizar el AFC se evaluó inicialmente el coeficiente de Mardia, que arrojó un valor de 43.09, considerado adecuado para el uso del método de estimación de máxima verosimilitud, por lo que se continuó el análisis con dicho método.

En la Tabla 2 se presentan los resultados ob- 
Tabla 3

Estadísticos descriptivos, consistencia interna, correlaciones en PTGI total y sus tres factores.

\begin{tabular}{|c|c|c|c|c|c|c|c|c|c|c|c|}
\hline \multirow[t]{2}{*}{ ESCALA } & \multicolumn{2}{|c|}{$\begin{array}{l}\text { Mujeres } \\
(\mathrm{n}=\mathbf{2 5 6})\end{array}$} & \multicolumn{2}{|c|}{$\begin{array}{l}\text { Hombres } \\
(\mathrm{n}=194)\end{array}$} & \multicolumn{2}{|c|}{$\begin{array}{c}\text { Total } \\
(\mathbf{N}=\mathbf{4 5 0})\end{array}$} & \multirow[b]{2}{*}{$\alpha$} & \multicolumn{4}{|c|}{ Correlaciones } \\
\hline & M & DE & M & DE & $\mathbf{M}$ & DE & & 1 & 2 & 3 & 4 \\
\hline 1. CPT-Autopercepción & 37.72 & 13.72 & 35.66 & 14.81 & 36.84 & 14.22 & 0.94 & na & $.84 * * *$ & $.70 * * *$ & $.97 * * *$ \\
\hline $\begin{array}{l}\text { 2. CPT-Relación } \\
\text { con otros }\end{array}$ & 22.98 & 7.93 & 20.69 & 8.92 & 21.99 & 8.44 & 0.91 & & na & $.72 * * *$ & $.93 * * *$ \\
\hline 3. CPT-Filosofía de vida & 6.00 & 3.04 & 5.09 & 3.11 & 5.61 & 3.10 & 0.83 & & & na & $.79 * * *$ \\
\hline 4. CPT Total & 66.70 & 23.03 & 61.44 & 25.31 & 64.44 & 24.15 & 0.96 & & & & na \\
\hline
\end{tabular}

Nota. ${ }^{* * *} p<.001 ;$ na $=$ no aplica.

tenidos en el AFC en los cinco modelos: a) modelo unifactorial; b) modelo jerárquico de tres factores de primer orden y un factor de segundo orden; c) modelo bifactor de tres factores específicos y un factor general; d) modelo jerárquico de cinco factores de primer orden y un factor de segundo orden, y e) modelo bifactor de cinco factores específicos y un factor general. En todos los casos el valor $\chi^{2}$ resultó estadísticamente significativo, lo que indica la existencia de una discrepancia entre los modelos propuestos y los datos analizados; sin embargo, dado el tamaño de la muestra, este resultado era esperable (Byrne, 2006).

Al analizar los estadísticos CFI, TLI y RMSEA se observa que el modelo de un factor mostró un ajuste insuficiente. Por su parte, los modelos bifactor de tres y cinco factores específicos obtuvieron valores aceptables casi equivalentes, incluso el valor del índice comparativo AIC.

Al analizar las correlaciones entre los factores se observaron diferencias entre el modelo de tres y cinco factores. En el modelo de cinco factores, las correlaciones entre los factores de apreciación de la vida, fuerza personal y prioridades de la vida estuvieron en el rango .96 a .98. En el modelo de tres factores, que supone los factores anteriores agrupados en una sola dimensión de cambios en la autopercepción, la más alta correlación observada fue de .84 entre ese factor y cambios en la relación con otros. Estas correlaciones encontradas en el modelo de cinco factores cuestionan su validez discriminante, ya que no son aceptables las correlaciones tan altas entre factores (Brown, 2006). A partir de este criterio, se estableció el modelo bifactor de tres factores específicos y un factor general como el más adecuado. En la Figura 1 se representa este modelo.

Al comparar hombres y mujeres, se observaron diferencias significativas en el PTGI total $\left(_{(448)}=2.30, p=.02\right)$ y obtuvieron mayores puntajes las mujeres que los hombres. A su vez, se observaron diferencias significativas de género tanto en el factor de cambio en la relación con otros $\left(t_{(448)}=2.89, p=.004\right)$ como en el factor de cambio en la filosofía de vida $\left(t_{(448)}=3.09, p=\right.$ .002), con mayores puntajes en el PTGI por parte de las mujeres. Las medias y desviaciones estándar de cada grupo se muestran en la Tabla 3.

Se procedió a evaluar la invarianza factorial del modelo entre hombres y mujeres. El análisis entre los grupos muestra la existencia de invarianza configural, pues los valores de los índices de ajuste RMSEA, TLI y CFI fueron aceptables. También muestra invarianza métrica, dado que el CFI no disminuyó su valor con respecto al modelo anterior y los otros índices casi no sufrieron 


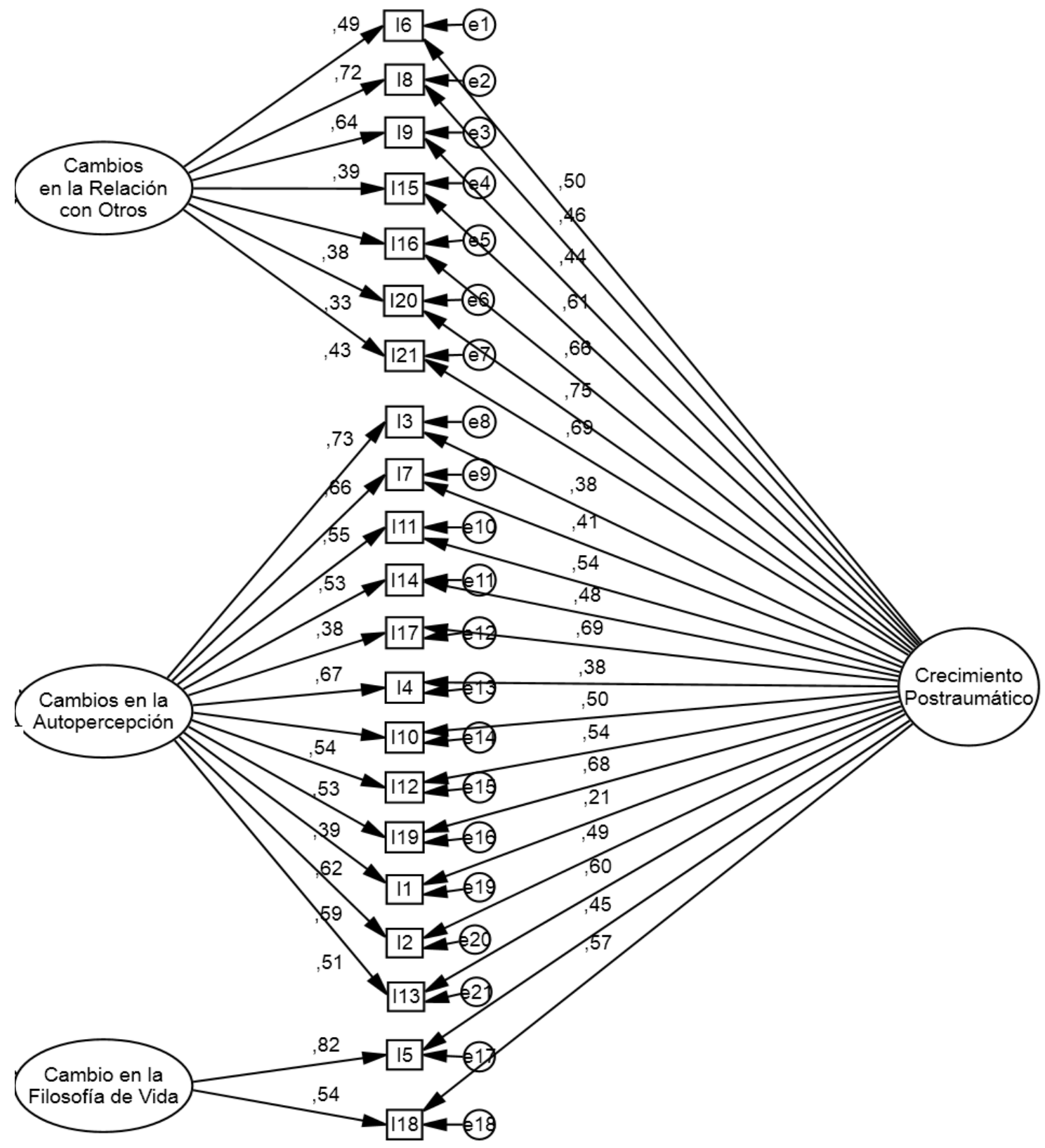

Figura 1

Modelo final de tres factores de primer orden y un factor general.

Nota. Los valores están estandarizados.

variaciones, por lo que se puede concluir que los dos grupos presentan cargas factoriales equivalentes. La invarianza fuerte también es aceptable pues los índices de ajuste siguen siendo apropiados y el CFI no disminuye, por lo que es posible concluir que los dos modelos evaluados son equivalentes respecto a los coeficientes factoria- les y a los interceptos. Finalmente, la invarianza estricta tampoco muestra una disminución en el CFI y presenta valores de ajuste aceptables en los otros índices, mostrando equivalencia en cargas factoriales, interceptos y errores. Con esto se ha aceptado el máximo nivel de invarianza al que se ha sometido el modelo (ver Tabla 4). 
Tabla 4

Índices de ajuste para la prueba de invarianza factorial entre hombres y mujeres.

\begin{tabular}{lcccccc}
\hline \multicolumn{1}{c}{ Modelos } & $\chi^{2}(\boldsymbol{g l})$ & $\boldsymbol{\Delta}^{2}$ & CFI & $\Delta$ CFI & TLI & RMSEA \\
\hline M1: configural & $817.59^{* * *}(370)$ & - & .93 & - & .92 & .05 \\
M2: métrica & $827.55^{* * *}(388)$ & -9.96 & .93 & .00 & .93 & .05 \\
M3: fuerte & $876.32^{* * *}(409)$ & -48.77 & .93 & .00 & .93 & .05 \\
M4: estricta & $907.88^{* * *}(431)$ & -31.56 & .93 & .00 & .93 & .05 \\
\hline
\end{tabular}

Nota. $\mathrm{M} 1=$ sin restricciones; $\mathrm{M} 2=\mathrm{M} 1+$ igualdad en cargas factoriales; $\mathrm{M} 3=\mathrm{M} 2+$ igualdad en los interceptos; M4 = M3 + igualdad en los errores o residuos. $* * * p<.001$.

Los valores del coeficiente alfa de Cronbach de la escala total y de sus factores son adecuados en el rango de .83 a .96 (ver Tabla 3). El rango de la correlación ítem-total corregida fue de .55 a .77 en la escala total, .57 a .77 en cambios en la autopercepción, .65 a .78 en cambios en la relación con otros y .70 en ambos ítems en cambios en la filosofía de vida.

En la Tabla 3 se presentan los estadísticos descriptivos del PTGI tanto para la escala total como para los tres factores específicos obtenidos en el AFC. También se pueden observar las correlaciones entre los distintos factores y la escala total. Al prorratear las medias de cada factor específico y dividir por el número de ítems de dicha subescala, se puede constatar que la dimensión de mayor crecimiento en esta población es la de cambio en la relación con otros (3.14), seguida por la de cambio en la autopercepción (3.07) y finalmente la de cambio en la filosofía de la vida (2.80).

\section{Discusión}

En la mayoría de los estudios en los que se ha utilizado AFC para evaluar la estructura factorial del PTGI, se ha observado un modelo de cinco factores similar al del estudio original de
Tedeschi y Calhoun (1996); sin embargo, también se han encontrado modelos de uno y de tres factores.

El presente estudio muestra que el modelo de un factor obtiene un ajuste insuficiente, mientras que los modelos jerárquicos de tres y cinco factores de primer orden y un factor general obtienen valores aceptables casi equivalentes; sin embargo el modelo bifactor de tres factores específicos y un factor general fue el que presentó mejor ajuste a los datos y mayor validez discriminante entre sus distintos factores, por lo que se consideró como el más adecuado. La solución bifactor con un factor general de CPT es concordante con la idea de un modelo teórico subyacente común de CPT (Tedeschi \& Calhoun, 1996). Este resultado coincide con lo encontrado por Rodríguez-Rey et al. (2016) y Garrido-Hernansaiz et al. (2017) en población española utilizando una versión más breve del instrumento.

En este modelo, el primer factor específico corresponde a cambios en la autopercepción, el segundo a cambios en la relación con otros y el tercero a cambios en la filosofía de vida, lo que coincide con la propuesta teórica original de Tedeschi y Calhoun (1996), quienes plantearon tres componentes del CPT. Además el presente estudio considera la presencia de un factor general, dando respaldo para considerar al CPT como 
un constructo que se puede evaluar tanto a través de sus dimensiones como a través de un puntaje único.

En el análisis de su confiabilidad, la escala mostró una alta consistencia interna tanto a nivel global como en cada uno de sus tres factores. Las correlaciones ítem-total corregidas tanto para la escala total como para los tres factores resultaron adecuadas. Ningún ítem mostró un comportamiento inadecuado.

En este estudio se encontraron diferencias en el CPT entre hombres y mujeres, lo que coincide con lo observado en diversos estudios y en el metaanálisis de Vishnevsky et al. (2010). Se ha planteado como una posible explicación a esta diferencia que las mujeres mantienen un nivel mayor de rumiación de pensamiento en comparación con los hombres tras vivir un hecho estresante, lo que les lleva a experimentar un mayor nivel de sufrimiento y evidencia una mayor prevalencia de estrés postraumático y depresión; pero a su vez, es este mismo sufrimiento el que les permite aprender a medida que se enfrentan a la experiencia (Avilés-Maldonado, Cova-Solar, Bustos, \& García, 2014; Vishnevsky et al., 2010).

Se realizó un análisis de la invarianza factorial del modelo comparando hombres y mujeres. Este análisis mostró la existencia de los tres niveles de invarianza evaluados, lo que muestra que los dos grupos presentan cargas factoriales, interceptos y errores equivalentes. Estos resultados permitieron aceptar el máximo nivel de invarianza al que fue sometido el modelo, posibilitando asumir que no existe sesgo por sexo en la escala y que efectivamente existe una diferencia en los niveles de CPT entre ambos grupos.

Dentro del análisis descriptivo se encontró que los sobrevivientes al terremoto del 16 de abril de 2016 mostraron un menor crecimiento en el factor de cambio en la filosofía de vida en relación con los otros factores; este fenómeno se podría explicar considerando que la muestra pertenece a la zona costera de Ecuador, un sector de bajo desarrollo religioso y espiritual en comparación con la zona andina. De la misma forma, los sobrevivientes ecuatorianos presentaron un mayor crecimiento en la dimensión de cambios en la relación con otros, fenómeno que se podría explicar por el tipo de sociedad colectivista de los ecuatorianos.

Comparado con una muestra de sobrevivientes chilenos al terremoto del año 2010 del mismo rango de edad (García, Jaramillo, et al., 2014), los puntajes de los participantes ecuatorianos fueron más bajos tanto en sus factores de cambios en la relación con otros y cambios en la autopercepción como en el puntaje total; sin embargo, el estudio chileno se produjo a los dos años del sismo; en cambio, el presente estudio se realizó doce meses después, por lo que es probable que dicho crecimiento siga aumentando en la medida que las personas tomen distancia suficiente del evento como para considerar áreas de crecimiento a partir de este. Estos resultados deberían ser contrastados con nuevas investigaciones en el campo del CPT para explicar este tipo de comportamiento.

Las limitaciones de este estudio radican en primer lugar en su diseño transversal que impide evaluar la estabilidad temporal de la escala. En segundo término, el muestreo intencionado utilizado puede sugerir que el grupo participante no necesariamente es representativo de la población general del Ecuador. Por último, la no inclusión de otros instrumentos impidió dar un valor discriminante e incluso predictivo a la variable CPT. Surge en consecuencia la posibilidad, al contar ahora con un instrumento psicométricamente adecuado para evaluar el CPT, de realizar estudios que relacionen el crecimiento con otras variables que se han observado relevantes como predictores de la misma. De este modo, sería posible llevar a 
cabo otros estudios con población latinoamericana expuesta a un desastre natural, y contrastar los datos obtenidos sobre CPT con variables como la religiosidad (García, Páez-Rovira, Zurtia, Martel, \& Reyes, 2014) y el uso de estrategias de afrontamiento (García, Cova, Rincón, Vázquez, \& Páez, 2016).

Contar con un instrumento que evalúe la posibilidad de cambios positivos tras vivir un evento adverso abre oportunidades para continuar investigando las secuelas de estos eventos más allá de las respuestas psicopatológicas en las que se ha centrado la investigación en las últimas décadas.

El presente estudio mostró que este instrumento cuenta con propiedades psicométricas apropiadas, lo que implica una alta confiabilidad, una estructura factorial de tres factores específicos y un factor general e invarianza en la medida entre hombres y mujeres, lo que asegura un instrumento no sesgado de acuerdo al sexo. Ante esto, podemos concluir que el PTGI es un instrumento útil para investigar el crecimiento postraumático en población ecuatoriana.

A la vez, esta adaptación permitirá guiar de mejor manera la intervención clínica post desastre al proporcionar una meta objetiva de trabajo terapéutico.

El análisis del CPT en la población afectada tendrá un gran impacto en la salud pública del país, pues permitirá predecir las probabilidades de presencia de respuestas psicopatológicas a largo plazo y desarrollar planes de promoción de la salud mental y prevención.

Por último, la adaptación de este instrumento psicométrico permite sentar una base empírica para la mejora en la gestión de los riesgos naturales centrada en la promoción de una salud mental positiva y en la posibilidad de que una catástrofe pueda ser también una oportunidad de crecimiento personal y colectivo. Esta intervención puede encaminarse hacia la promoción de fortalezas del carácter y de estrategias de afrontamiento activas alentando la cercanía con la familia y otras redes sociales junto con la generación de mayor valoración de la propia existencia.

\section{Referencias}

American Psychiatric Association (2013). Manual Diagnóstico y Estadístico los Trastornos Mentales DSM-V. Barcelona, España: Masson.

Anderson Jr, W. P., \& Lopez-Baez, S. I. (2008). Measuring growth with the Posttraumatic Growth Inventory. Measurement and Evaluation in Counseling and Development, 40(4), 215-227.

Arbuckle, J. L. (2009). Amos (Version 20.0) [Computer Program]. Chicago, IL: IBM SPSS.

Avilés-Maldonado, P., Cova-Solar, F., Bustos, C., \& García, F. E. (2014). Afrontamiento y rumiación frente a eventos adversos y crecimiento postraumático en estudiantes universitarios. Liberabit, 20(2), 281-292. Recuperado de http://www.scielo.org.pe/pdf/liber/ v20n2/a09v20n2.pdf

Bentler, P. (2005). EQS Structural equations program manual. Encino, CA: Multivariate Software.

Bollen, K. A. (1989). Structural equations with latent variables. New York, NY: John Wiley \& Sons. doi: 10.1002/9781118619179

Brown, T. A. (2006). Confirmatory Factor Analysis for Applied Research. New York, NY: Guilford Press.

Brunet, J., McDonough, M. H., Hadd, V., Crocker, P. R. E., \& Sabiston, C. M. (2010). The Posttraumatic Growth Inventory: An examination of the factor structure and invariance among breast cancer survivors. PsychoOncology, 19(8), 830-838. doi: 10.1002/pon.1640

Byrne, B. (2006). Structural equation modeling with Mplus. New York, NY: Routledge.

Calhoun, L. G., Cann, A., \& Tedeschi, R. G. (2010). The Posttraumatic Growth Model: Sociocultural considerations. En T. Weiss \& R. Berger (Eds.), Posttraumatic growth and culturally competent 
practice: Lessons learned from around the globe (pp.1-14). New York, NY: John Wiley \& Sons. doi: 10.1002/9781118270028.ch1

Cheung, G. W., \& Rensvold, R. B. (2002). Evaluating goodness-of-fit indexes for testing measurement invariance. Structural Equation Modeling, 9(2), 233255. doi: 10.1207/S15328007SEM0902_5

Costa-Requena, G., \& Moncayo, F. L. G. (2007). Crecimiento postraumático en pacientes oncológicos. Análisis y Modificación de Conducta, 33(148), 229-250. Recuperado de http://uhu.es/publicaciones/ ojs/index.php/amc/article/view/1215

Elosúa, P. (2005). Evaluación progresiva de la invarianza factorial entre las versiones original y adaptada de una escala de autoconcepto. Psicothema, 17(2), 356362. Recuperado de: http://www.ehu.eus/gip/publicaciones/articulos/2005/2.pdf

Esparza-Baigorria, T., Leibovich de Figueroa, N., \& Martínez-Terrer, M. (2016). Propiedades psicométricas del Inventario de Crecimiento Postraumático en pacientes oncológicos en población argentina. Ansiedad y Estrés 22(2) 97-103. doi: 10.1016/j. anyes.2016.06.002

García, F. E., Capponi, C., Hinrichs, F., Lillo, F., Rodríguez, C., \& Sánchez, J. (2016). Violencia policial y afrontamiento: Crecimiento postraumático en pobladores de una comunidad aislada del sur de Chile. Universitas Psychologica, 15(4). doi: 10.11144/ Javeriana.upsy15-4.vpac

García, F. E., Cova-Solar, F., \& Melipillán, R. (2013). Propiedades psicométricas del Inventario de Crecimiento Postraumático en población Chilena afectada por un desastre natural. Revista Mexicana de Psicología, 30(2), 143-151. Recuperado de http:// www.redalyc.org/articulo.oa?id=243033029007http://www.redalyc.org/articulo.oa?id=243033029007

García, F. E., Cova, F., Rincón, P., Vázquez, C., \& Páez, D. (2016). Coping, rumination and posttraumatic growth in people affected by an earthquake. Psicothema, 28(1), 59-65. doi: 10.7334/psicothema2015.100

García, F. E., Jaramillo, C., Martínez, A. M., Valenzuela,
I., \& Cova-Solar, F. (2014). Respuestas psicológicas ante un desastre natural: Estrés y crecimiento postraumático. Liberabit, 20, 121-130. Recuperado de http://www.scielo.org.pe/pdf/liber/v20n1/a11v20n1. pdf

García, F. E., Páez, D., Reyes-Reyes, A., \& Álvarez, R. (2017). Religious coping as moderator of psychological responses to stressful events: A longitudinal study. Religions, 8(62), 1-13. doi: 10.3390/ rel 8040062

García, F. E., Páez-Rovira, D., Zurtia, G. C., Martel, H. N, \& Reyes, A. R. (2014). Religious coping, social support and subjective severity as predictors of posttraumatic growth in people affected by the earthquake in Chile on 27/2/2010. Religions, 5(4), 1132-1145. doi: 10.3390/rel5041132

Garcia da Silva, T., Candia-Donat, J., Gauer, G., \& HaagKristensen, C. (2016). Posttraumatic growth measures: Translation and adaptation of three self-report instruments to Brazilian Portuguese. Archives of Clinical Psychiatry (Sao Paulo), 43(3), 47-50. doi: 10.1590/0101-60830000000083

Garrido-Hernansaiz, H., Rodríguez-Rey, R., \& Alonso-Tapia, J. (2017). Posttraumatic Growth Inventory: Factor structure in Spanish-speaking people living with HIV. AIDS Care, 1-4. doi: 10.1080/09540121.2017.1291900

Hooper, L. M., Marotta, S. A., \& Depuy, V. (2009). A confirmatory factor analytic study of the Posttraumatic Growth Inventory among a sample of racially diverse college students. Journal of Mental Health, 18(4), 335-343. doi: 10.1080/09638230802522502

IBM Corp (2011). IBM SPSS Statistics for Windows, Version 21.0. Armonk, NY: IBM Corp.

Joseph, S., \& Linley, P. A. (2008). Psychological assessment of growth following adversity: A review. En S. Joseph \& P. Linley (Eds.), Trauma, recovery, and growth: Positive psychological perspectives on posttraumatic stress (pp. 21-38). New York, NY: John Wiley \& Sons. doi: 10.1002/9781118269718.ch2

Joseph, S., Linley, P. A., \& Harris, G. J. (2004). Understanding 
positive change following trauma and adversity: A structural clarification. Journal of Loss and Trauma, 10(1), 83-96. doi: 10.1080/15325020490890741

Kline, R. B. (2005). Principles and practice of structural equation modeling (3th ed.). New York, NY: Guilford.

Konkolÿ-Thege, B., Kovács, É., \& Balog, P. (2014). A bifactor model of the Posttraumatic Growth Inventory. Health Psychology and Behavioral Medicine: An Open Access Journal, 2(1), 529-540. doi: $10.1080 / 21642850.2014 .905208$

Mardia, K. V. (1970). Measures of multivariate skewness and kurtosis with applications. Biometrika, 57(3), 519-530. doi: 10.2307/2334770

Mardia, K. V. (1974). Applications of some measures of multivariate skewness and kurtosis in testing normality and robustness studies. Sankhya: The Indian Journal of Statistic, 36(2), 115-128. Recuperado de http://www.jstor.org/stable/25051892

Martínez-Arias, R. (1995). Psicometría: teoría de los test psicológicos y educativos. Madrid, España: Síntesis.

Palmer, G. A., Graca, J. J., \& Occhietti, K. E. (2012). Confirmatory factor analysis of the Posttraumatic Growth Inventory in a veteran sample with posttraumatic stress disorder. Journal of Loss and Trauma, 17(6), 545-556. doi: 10.1080/15325024.2012.678779

Powell, S., Rosner, R., Butollo, W., Tedeschi, R. G., \& Calhoun, L. G. (2003). Posttraumatic growth after war: A study with former refugees and displaced people in Sarajevo. Journal of Clinical Psychology, 59(1), 71-83. doi: 10.1002/jclp.10117

Rodríguez-Rey, R., Alonso-Tapia, J., Kassam-Adams, N., $\&$ Garrido-Hernansaiz, H. (2016). The factor structure of the Posttraumatic Growth Inventory in parents of critically ill children. Psicothema, 28(4), 495-503. doi: 10.7334/psicothema2016.162

Rodríguez-Ayán, M. N., \& Ruiz Diaz, M. Á. (2008). Atenuación de la asimetría y de la curtosis de las puntuaciones observadas mediante transformaciones de variables: Incidencia sobre la estructura factorial. Psicológica: Revista de Metodología y Psicología
Experimental, 29(2), 205-228. Recuperado de http:// www.uv.es/revispsi/articulos2.08/6RODRIGUEZ. pdf

Taku, K., Cann, A., Calhoun, L. G., \& Tedeschi, R. G. (2008). The factor structure of the Posttraumatic Growth Inventory: A comparison of five models using confirmatory factor analysis. Journal of Traumatic Stress, 21(2), 158-164. doi: 10.1002/jts.20305

Tedeschi, R. G., \& Calhoun, L. G. (1996). The Posttraumatic Growth Inventory: Measuring the positive legacy of trauma. Journal of Traumatic Stress, 9(3), 455-471. doi: 10.1002/jts.2490090305

Tedeschi, R. G., \& Calhoun, L. G. (2000). Posttraumatic growth: A new focus in psycho traumatology. Psytalk, Newsletter of the British Psychological Society Student Members Group, April 2000.

Tedeschi, R. G., \& Calhoun, L. G. (2008). Crecimiento postraumático en las intervenciones clínicas cognitivo-conductuales. En V. Caballo (Ed). Manual para el tratamiento cognitivo-conductual de los trastornos psicológicos (pp. 30-49). Madrid, España: Siglo XXI.

Vishnevsky, T., Cann, A., Calhoun, L. G., Tedeschi, R. G., \& Demakis, G. J. (2010). Gender differences in self-reported posttraumatic growth: A meta-analysis. Psychology of Women Quarterly, 34(1), 110-120. doi: 10.1111/j.1471-6402.2009.01546.x

Weiss, T., \& Berger, R. (2006). Reliability and validity of a Spanish version of the Posttraumatic Growth Inventory. Research on Social Work Practice, 16(2), 191-199. doi: 10.1177/1049731505281374 
Anexo

Inventario de Crecimiento Postraumático

Sexo: F ( ) M ( ) Edad:

Fecha:

De acuerdo a la escala mostrada a continuación, indique cuánto cambio tuvo en su vida a raíz de la crisis/ desastre vivida. Use la siguiente escala:

0 : No he experimentado ningún cambio en mi vida a raíz de la crisis/desastre

1: Ha habido un cambio minúsculo en mi vida a raíz de la crisis/desastre

2: Ha habido un pequeño cambio en mi vida a raíz de la crisis/desastre

3: Ha habido un cambio moderado en mi vida a raíz de la crisis/desastre

4: Ha habido un gran cambio en mi vida a raíz de la crisis/desastre

5: Ha habido un cambio enorme en mi vida a raíz de la crisis/desastre

\begin{tabular}{|c|c|c|c|c|c|c|c|}
\hline & Posibles áreas de crecimiento y cambio & $\mathbf{0}$ & 1 & 2 & 3 & 4 & 5 \\
\hline 1 & Han cambiado mis prioridades acerca de lo que es importante en mi vida. & & & & & & \\
\hline 2 & Tengo una mejor apreciación acerca del valor de mi propia vida. & & & & & & \\
\hline 3 & Desarrollé nuevos intereses. & & & & & & \\
\hline 4 & Tengo un mejor sentido de autosuficiencia. & & & & & & \\
\hline 5 & Soy una persona más espiritual, he crecido espiritualmente. & & & & & & \\
\hline 6 & $\begin{array}{l}\text { Puedo apreciar más claramente que puedo contar } \\
\text { con los demás en tiempos difíciles. }\end{array}$ & & & & & & \\
\hline 7 & He construido un nuevo rumbo o caminos de vida. & & & & & & \\
\hline 8 & $\begin{array}{l}\text { Siento un mayor sentido de proximidad con las } \\
\text { personas o mayor cercanía hacia los demás. }\end{array}$ & & & & & & \\
\hline 9 & Estoy más dispuesto a expresar mis emociones. & & & & & & \\
\hline 10 & Siento que puedo arreglármelas mejor en los momentos difíciles. & & & & & & \\
\hline 11 & Puedo hacer mejores cosas con mi vida. & & & & & & \\
\hline 12 & Estoy más dispuesto a aceptar la forma en que las cosas se dan. & & & & & & \\
\hline 13 & Puedo apreciar mejor cada día. & & & & & & \\
\hline 14 & Hay nuevas oportunidades que antes no estaban disponibles. & & & & & & \\
\hline 15 & Siento más compasión por los demás. & & & & & & \\
\hline 16 & Pongo más esfuerzo en mis relaciones. & & & & & & \\
\hline 17 & Estoy más dispuesto a intentar cambiar las cosas que necesitan ser cambiadas. & & & & & & \\
\hline 18 & Tengo o he desarrollado una mayor fortaleza religiosa. & & & & & & \\
\hline 19 & He descubierto que era o soy más fuerte de lo que pensaba. & & & & & & \\
\hline 20 & $\begin{array}{l}\text { He aprendido lo maravillosas que son las personas } \\
\text { o lo extraordinarias que pueden ser. }\end{array}$ & & & & & & \\
\hline 21 & Acepto de mejor manera el necesitar a los demás. & & & & & & \\
\hline
\end{tabular}

